Supporting Information

\title{
Tuned Chemical Bonding Ability of Au at Grain Boundaries for Enhanced Electrochemical $\mathrm{CO}_{2}$ Reduction
}

Kang-Sahn Kim ${ }^{1}$, Won June Kim ${ }^{1}$, Hyung-Kyu Lim², Eok Kyun Lee ${ }^{1, *}$, and Hyungjun Kim ${ }^{2, *}$

${ }^{1}$ Department of Chemistry, Korea Advanced Institute of Science and Technology (KAIST), Yuseong-gu, Daejeon 305-701, Korea

${ }^{2}$ Graduate School of Energy, Environment, Water, and Sustainability (EEWS), Korea Advanced Institute of Science and Technology (KAIST), Yuseong-gu, Daejeon 305-701, Korea 


\section{*Correspondence to E.K.L. (eklee@kaist.ac.kr) and H.K. (linus16@kaist.ac.kr)}

\section{Simulation details}

All calculations were performed using first-principles density functional theory (DFT) as implemented in the SIESTA code $^{1}$. The generalized-gradient approximation (GGA) exchange-correlation functional of Perdew, Burke, and Ernzerhof (PBE) was employed in the calculations $^{2}$. Numerical atomic orbitals $(\mathrm{NAOs})^{3}$ were used for the basis functions of the valence electrons, and the interactions between valence and core electrons were treated with norm-conserving scalar-relativitic pseudopotentials, including nonlinear partial-core corrections $^{4}$. Double- $\zeta$ plus single- $\zeta$ polarization basis functions were employed for Au, C, O, and $\mathrm{H}$ and were optimized to fit the experimental surface energy of the clean Au slab, experimental grain boundary formation energy of the Au bulk structure, and theoretical $\mathrm{COOH}$ and $\mathrm{CO}$ binding energies.

\section{A. The grain boundary modelling}

Of the many possible high-angle grain boundaries (HAGBs), the $\Sigma 3\{112\}$ symmetric tilt HAGB, which has the highest density of coincident lattice sites (CLSs) at the GB plane, was chosen because it has a very stable atomic structure and is the most abundant HAGB in the $\mathrm{Au}<111>$ texture based on experimental analysis of the grain boundary character distribution $(\mathrm{GBCD})^{5}$. The initial $\Sigma 3\{112\}$ GB structure on the Au (111) surface was constructed from the simple defect-free face-centered cubic (FCC) Au bulk structure. Specifically, the two bulk domains that form the bulk $\Sigma 3\{112\}$ HAGB structure were constructed by exposing a $\{112\}$ face of the FCC Au crystal and performing an inversion operation on the exposed $\{112\}$ face as plane of symmetry to obtain its mirror image. Before joining the two grains, the atomic positions and distances near the $\{112\}$ grain boundary must be handled in a delicate manner through trial and error to minimize grain boundary formation energy and to avoid unrealistic 
atomic ordering.

After constructing the bulk $\Sigma 3\{112\}$ HAGB structure, the $\Sigma 3\{112\}$ HAGB on the Au (111) surface was obtained by removing the top of the GB structure along the $\{111\}$ plane. To construct the unit cell with periodic boundary conditions (PBCs), the regularity and periodicity of the atomic layers in all three directions, i.e., the [112] direction, the [111] direction, and the direction normal to both [112] and [111], were examined. The initial structure was finally obtained by setting the [112] and [111] directions as the $y$ and $z$ axes, respectively, with certain repeat units of the regular atomic layers. This structure consisted of a 3-layered 8.952 $\AA$ × 43.390 $\AA$ × 7.309 $\AA$ orthorhombic unit cell with a $\Sigma 3\{112\}$ HAGB on the $\mathrm{Au}(111)$ surface. (Fig. S6a)

The stability of the structure was not considered in the mathematical operations; therefore, the initial structure of the $\Sigma 3\{112\}$ HAGB on the Au (111) surface had to be optimized. First, the geometry of the GB bulk model containing $150 \mathrm{Au}$ atoms $(8.952 \AA \times 43.390 \AA \times 7.309 \AA$ orthorhombic unit cell) was fully optimized until the atomic forces on the Au atoms were less than $0.01 \mathrm{eV} \cdot \AA^{-1}$ (Fig. S6b). Then, the GB slab model, which consisted of the fully relaxed 3layer GB bulk structure and a vacuum layer of $20 \AA(8.952 \AA \times 43.390 \AA \times 27.309 \AA$ orthorhombic supercell), was partially optimized keeping the middle and bottom atomic layers fixed (Fig. S6c).

Being GB a bulk defect, it causes substantial deformation in local atomic distribution and local symmetry. Therefore, the concave and convex structures are natural consequences of the stabilization of GB structures, and these are not artifacts due to the limited number of layers of the slab model.

The clean slab without a GB was modeled using the same procedure except that the $b$ 
parameter was $41.347 \AA$ and the structure contained 144 Au atoms. When a new adsorbate was placed on the surface, the geometries of the adsorbate molecule and top layer of the slab structure were optimized using the same force tolerance criterion.

\section{B. The binding free energy and the solvation energy}

Each component of the thermodynamic free energy was calculated according to the following procedure: the monatomic ideal gas, the rigid rotor and harmonic oscillator approximations were used to determine the translational, rotational, and vibrational components of the thermodynamic free energy from the molecular partition functions. The vibrational frequencies were evaluated by diagonalizing the partial Hessian matrix of the adsorbate molecule only.

The solvation energy was included in a non-self-consistent manner using the Delphi code ${ }^{6,7}$ due to the lack of an appropriate internal solvation module in the SIESTA program. Solvation effects were included for solvated species, solvated bound species, and solvated metal surface to estimate the overall solvation effect in the binding process. Estimation of each species were done employing Mulliken charges and atomic coordinates for each atom (including Au atom) in gas-phase optimized supercell and their scaled van der Waals radii to solve the Poisson-Boltzmann equation. Since the SIESTA program cannot include the solvation effect in a self-consistent manner, unlike the VASP program where the self-consistent reaction field (SCRF) calculation is available, we adopted following non self-consistent procedure;

1. Obtain gas-phase optimized structure and Mulliken charges from DFT.

2. Set the atomic radius of each atom.

3. Compute the solvation free energy by solving the Poisson-Boltzmann equation using Delphi code. Delphi code reads the atomic positions, atomic 
charges, and atomic radii (all of which were determined in previous steps).

Although atomic positions and atomic charges can be rather unambiguously determined from DFT results, there exists a certain degree of ambiguity in determining the atomic radii. When we adopt the well-known van der Waal's radii as listed in Table S6, the COOH and CO binding free energies for the GB-free clean surface in aqueous environment were calculated as $-1.420 \mathrm{eV}$ and $-0.397 \mathrm{eV}$, respectively. These values are in consistent with $-1.310 \mathrm{eV}$ and $0.310 \mathrm{eV}$ obtained from VASP + self-consistent solvation calculations, showing reasonable accuracy of our current solvation calculation method. By scaling the van der Waal's radii of $\mathrm{C} / \mathrm{O} / \mathrm{H}$ by 0.6 , we further improved the contribution of solvation effect in the binding free energies, which results in $-1.359 \mathrm{eV}$ and $-0.380 \mathrm{eV}$ for $\mathrm{COOH}$ and $\mathrm{CO}$, respectively (from reference 8) (Table S7). 
Figures and Tables

(a)

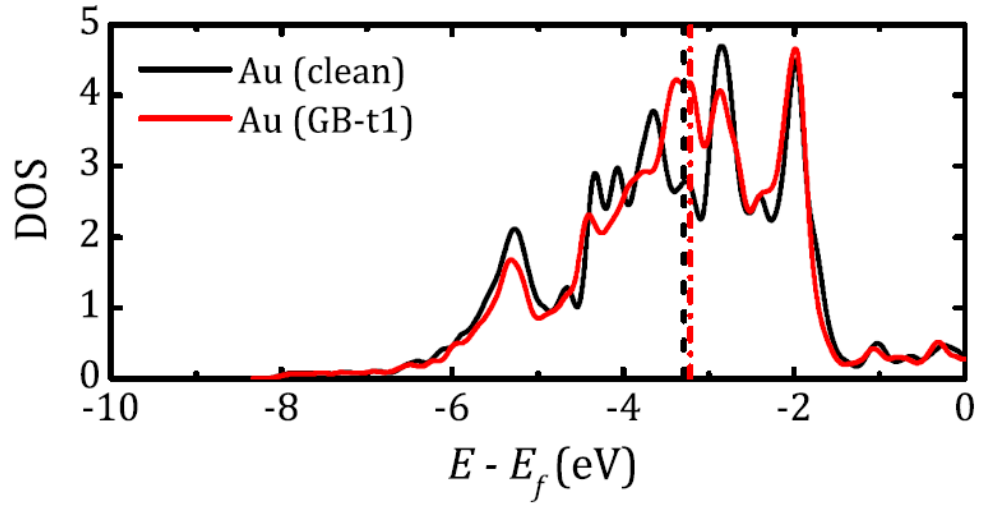

(b)

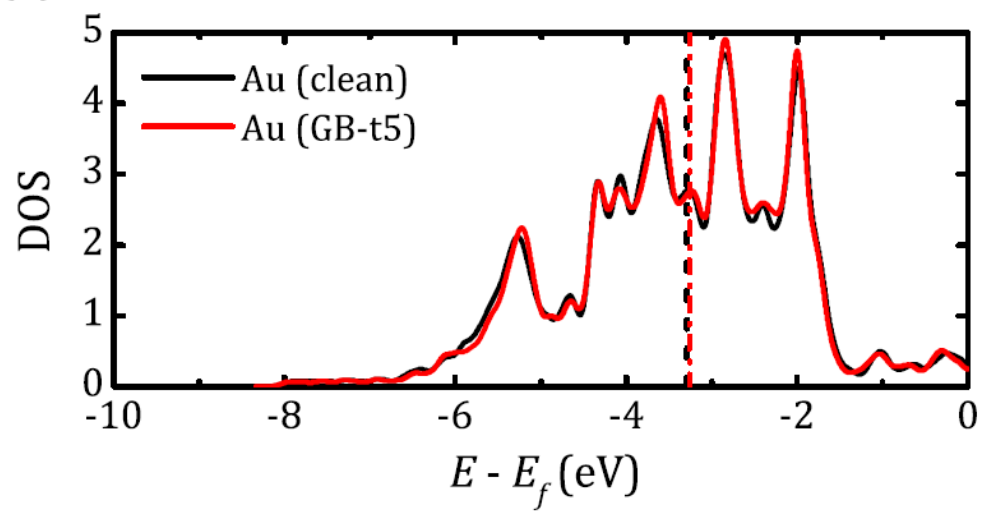

Figure S1. Au density of states (DOS) at the (a) t1 site (near the GB) and (b) t5 site (bulk regime) compared to the Au DOS at the clean Au (111) surface. The location of the $d$-band center is indicated by vertical dashed and dash-dotted lines for the clean and GB surfaces, respectively. 

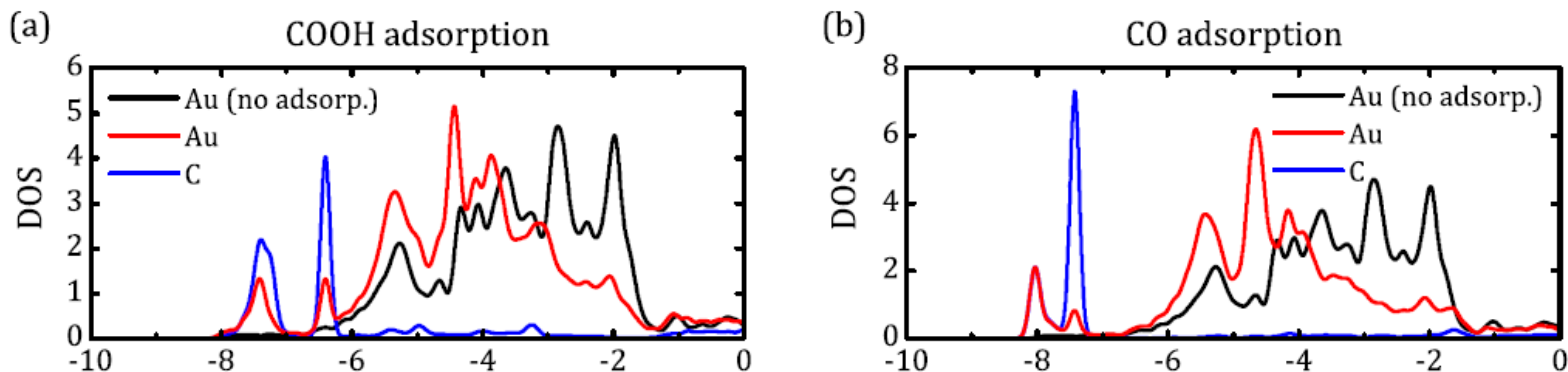

(c)

(d)
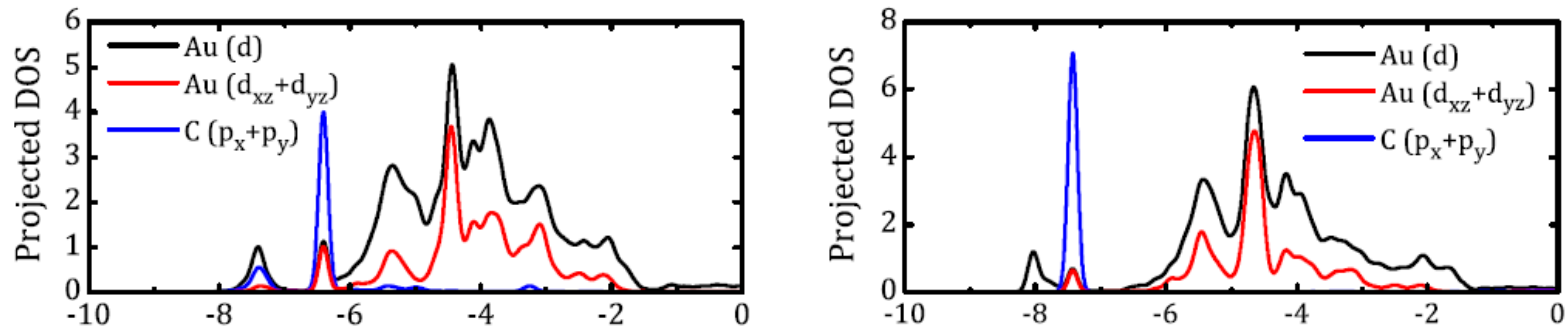

(e)

(f)
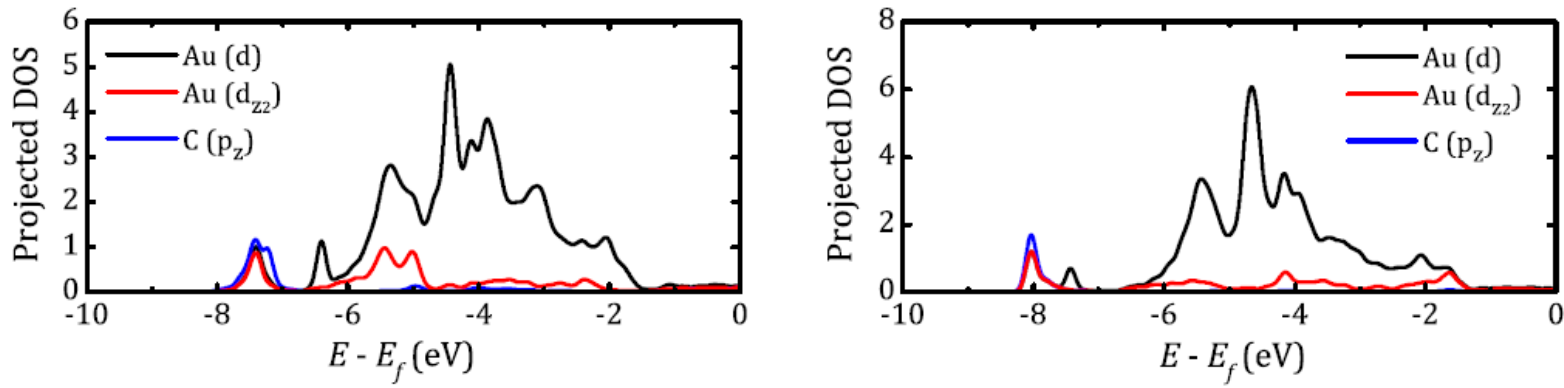

Figure S2. Au density of states (DOS) when (a, c, e) $\mathrm{COOH}$ and (b, d, f) $\mathrm{CO}$ are adsorbed on the clean $\mathrm{Au}$ (111) surface. (a) and (b) Au DOS before (black) and after (red) adsorption. The DOS of the $\mathrm{COOH} / \mathrm{CO} \mathrm{C}$ atom is also shown in blue. As shown by the projected density of states (PDOS) in (c)-(f), the total Au DOS in this energy range is mainly due to the Au $d$-band, and the two sharp peaks that appear after adsorbate binding are due to $d_{z 2}-p_{z}$ (lower-energy peak) and $d_{x z / y z}-p_{x / y}$ (higher-energy peak) interactions. 
(a) $\mathrm{COOH}$ adsorption

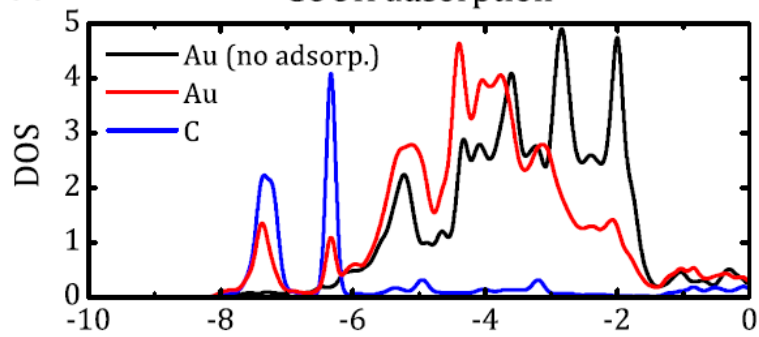

(c)

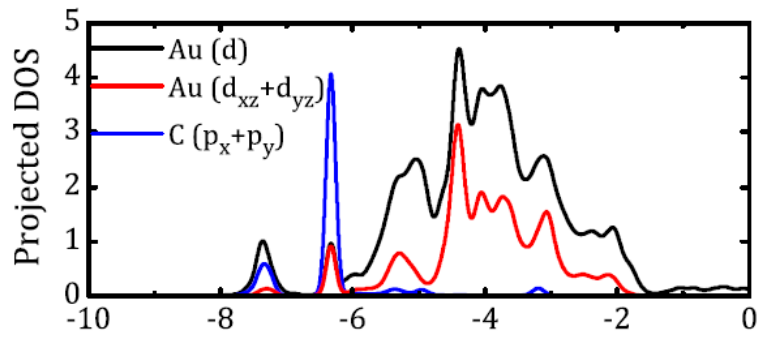

(e)

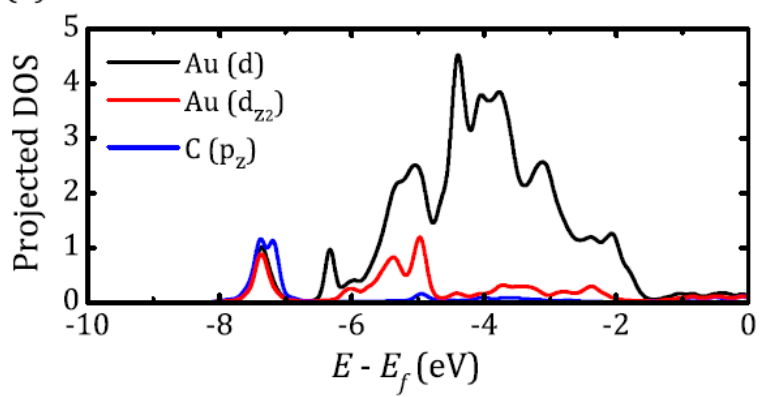

(b)

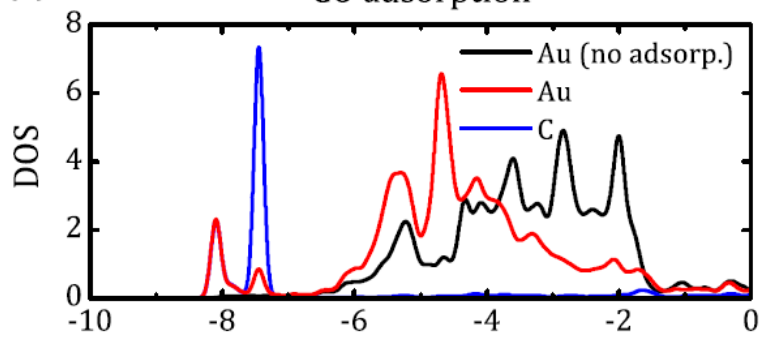

(d)

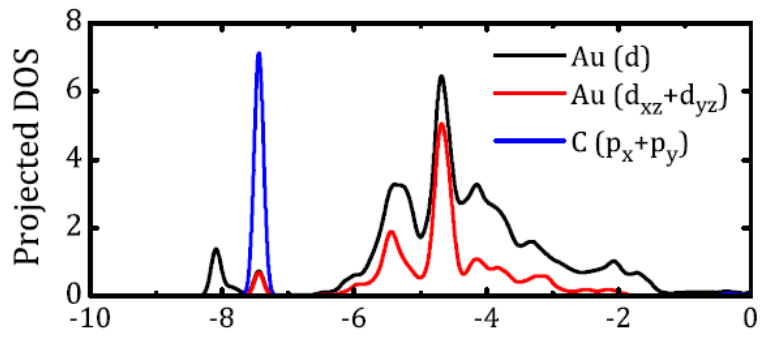

(f)

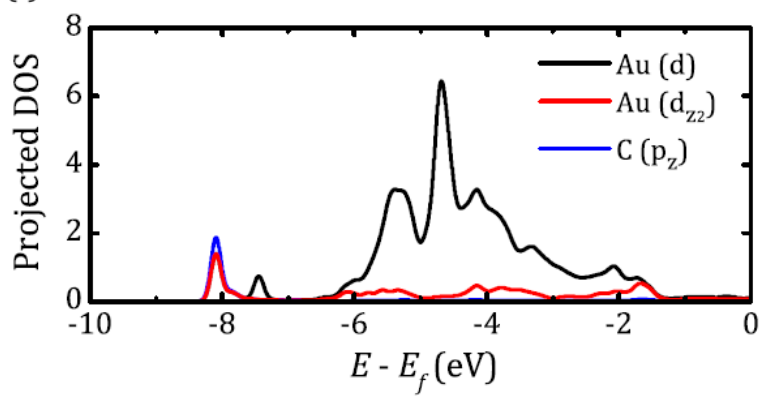

Figure S3. Au density of states (DOS) when (a, c, e) $\mathrm{COOH}$ and (b, d, f) $\mathrm{CO}$ are adsorbed at the $t 5$ site in the middle of the two grain boundaries (bulk regime). (a) and (b) Au DOS at the t5 site before (black) and after (red) adsorption. The DOS of the $\mathrm{COOH} / \mathrm{CO} \mathrm{C}$ atom is also shown in blue. As shown by the projected density of states (PDOS) in (c)-(f), the total Au DOS in this energy range is mainly due to the Au $d$-band, and the two sharp peaks that appear after adsorbate binding are due to $d_{z 2}-p_{z}$ (lower-energy peak) and $d_{x z / y z}-p_{x / y}$ (higher-energy peak) interactions. 

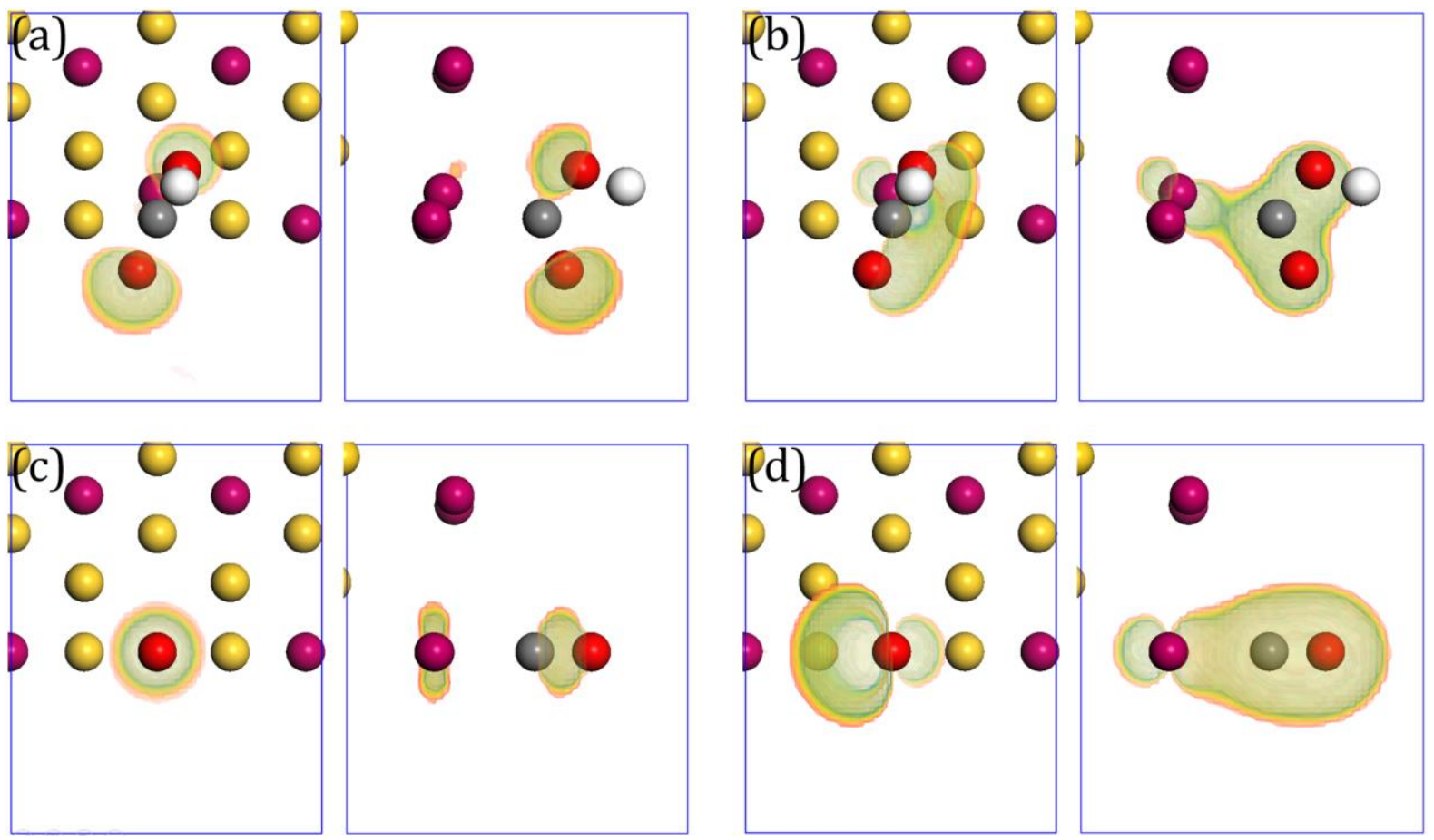

Figure S4. Wavefunction density maps (top and side views) of the localized states that appear as two sharp peaks in the DOS in Fig. 3. Localized states corresponding to the (a) lowerenergy and (b) higher-energy peaks induced by $\mathrm{COOH}$ adsorption. Localized states corresponding to the (c) lower-energy and (d) higher-energy peaks induced by $\mathrm{CO}$ adsorption. Based on the wavefunction symmetry and nodal plane orientation, the lower-energy and higher-energy states correspond to the adsorbate-to-metal $\sigma$-bonding and metal-to-ligand $\pi$ backbonding states, respectively. 
(a) $\mathrm{COOH}$

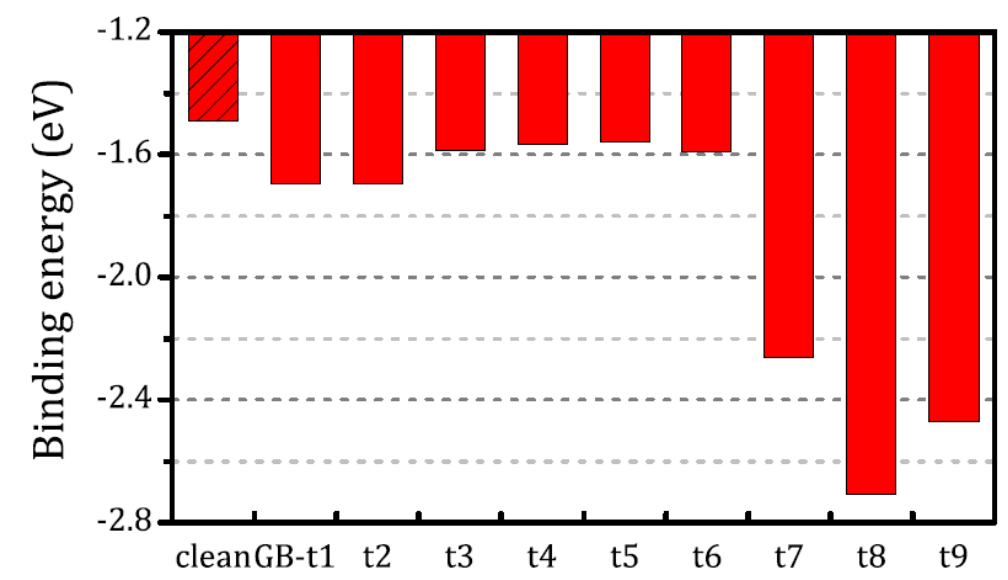

(b) CO

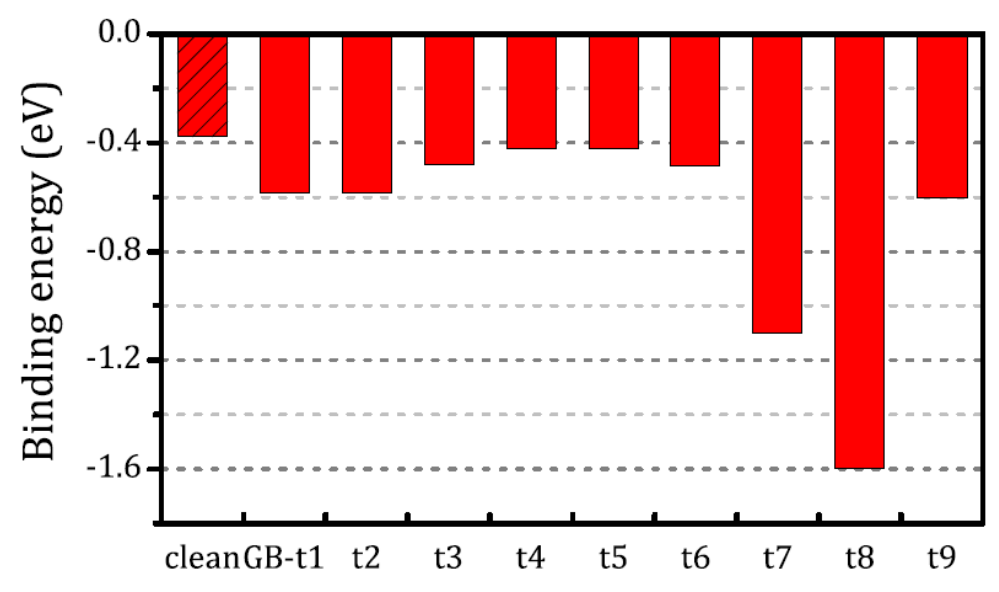

Figure S5. Site-dependent binding energies for (a) $\mathrm{COOH}$ and (b) $\mathrm{CO}$ adsorption. 
(a)

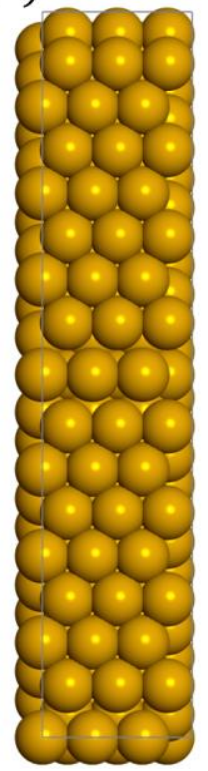

(b)

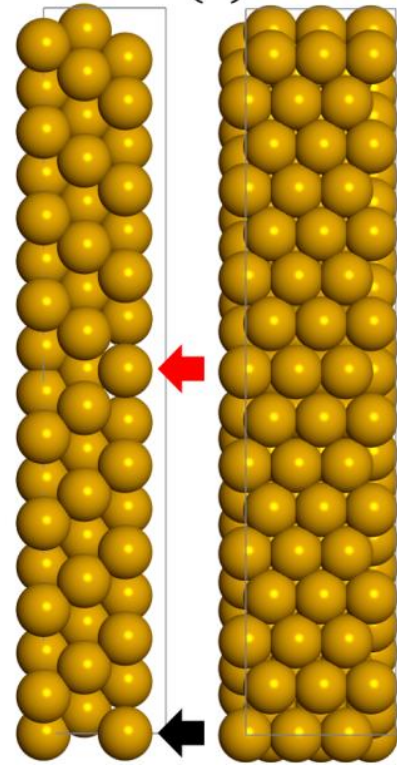

(c)

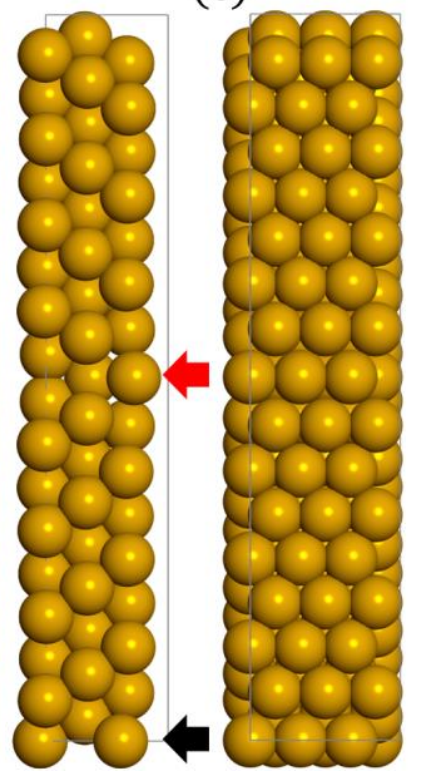

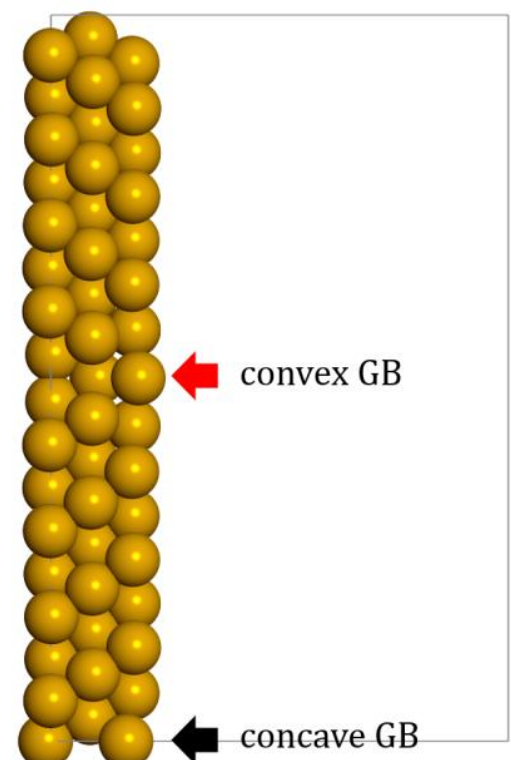

Figure S6. Top and side views of (a) GB bulk model (initial structure), (b) GB bulk model (after geometry optimization with cell relaxation), and (c) GB slab model (after geometry optimization). Convex GB and concave GB are indicated with red and black arrows, respectively.

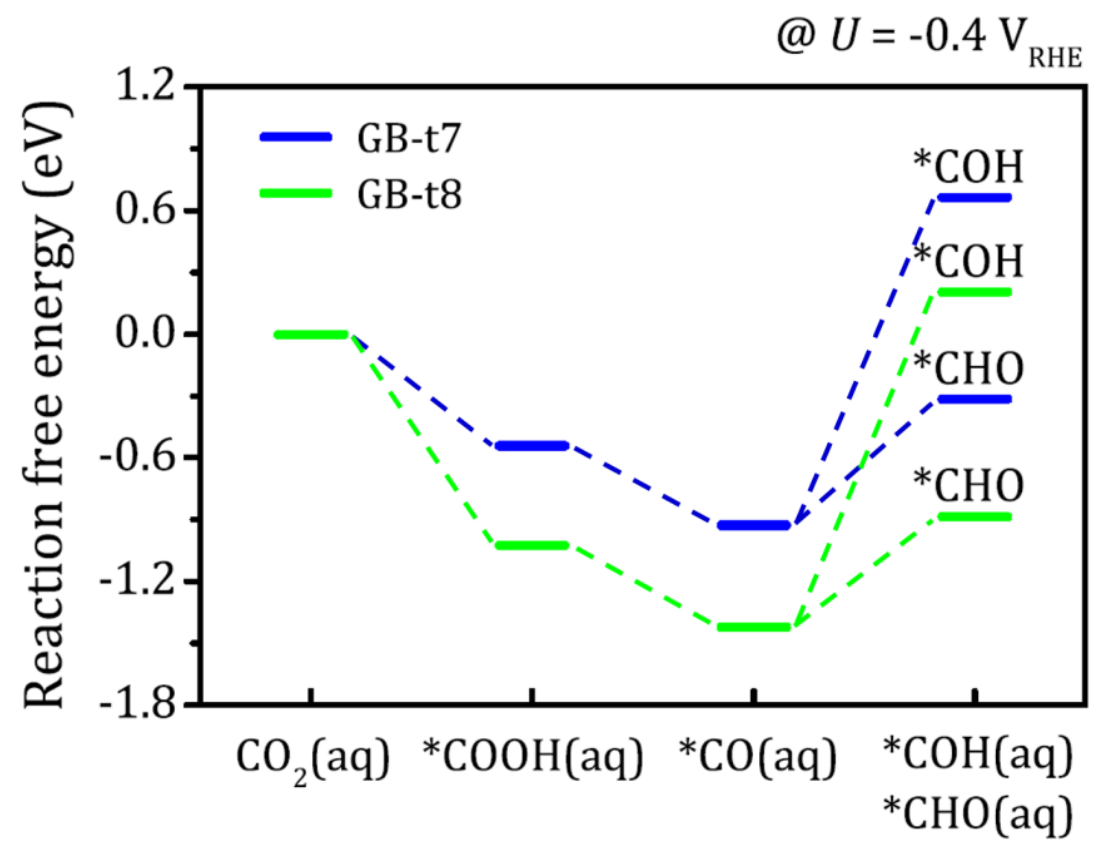

Figure S7. Reaction free energy profiles for the $3^{\text {rd }}$ reduction step of $* \mathrm{CO}$ at $\mathrm{t} 7$ (blue) and $\mathrm{t} 8$ (green) sites under a finite bias potential of $U=-0.4 \mathrm{~V}_{\mathrm{RHE}}$. 
Table S1. Occupations of the $\sigma$-bonding and $\pi$-backbonding states calculated by integrating the localized DOS peaks in Fig. 3.

\begin{tabular}{|c|c|c|c|c|c|c|c|c|c|c|c|}
\hline & & Clean & GB-t1 & GB-t2 & GB-t3 & GB-t4 & GB-t5 & GB-t6 & GB-t7 & GB-t8 & GB-t9 \\
\hline \multirow{2}{*}{$\begin{array}{c}\sigma \text {-bonding } \\
\text { (peak 1) }\end{array}$} & $\mathrm{COOH}$ & 0.720 & 0.715 & 0.715 & 0.717 & 0.722 & 0.720 & 0.722 & 0.712 & 0.714 & 0.711 \\
\hline & $\mathrm{CO}$ & 0.415 & 0.436 & 0.424 & 0.425 & 0.422 & 0.422 & 0.418 & 0.422 & 0.423 & 0.431 \\
\hline \multirow{2}{*}{$\begin{array}{c}\pi \text {-backbonding } \\
(\text { peak } 2)\end{array}$} & $\mathrm{COOH}$ & 0.907 & 0.948 & 0.939 & 0.917 & 0.908 & 0.908 & 0.920 & 0.990 & 0.979 & 0.999 \\
\hline & $\mathrm{CO}$ & 1.371 & 1.373 & 1.370 & 1.363 & 1.365 & 1.366 & 1.374 & 1.360 & 1.363 & 1.361 \\
\hline
\end{tabular}

Table S2. Average nearest neighbor atomic distances and their corresponding standard deviations $(\AA)$.

\begin{tabular}{cccccccccccc}
\hline & & Clean & GB-t1 & GB-t2 & GB-t3 & GB-t4 & GB-t5 & GB-t6 & GB-t7 & GB-t8 & GB-t9 \\
\hline \hline Top layer NN & AV & 2.984 & 3.172 & 3.115 & 3.038 & 3.010 & 3.003 & 3.010 & 3.038 & 3.111 & 3.163 \\
& STD & 0.000 & 0.145 & 0.127 & 0.050 & 0.022 & 0.014 & 0.023 & 0.050 & 0.121 & 0.139 \\
\hline \multirow{2}{*}{ 2nd layer NN } & AV & 2.984 & 2.971 & 2.950 & 2.977 & 2.984 & 2.987 & 2.985 & 2.984 & 3.024 & 3.274 \\
& STD & 0.000 & 0.000 & 0.045 & 0.057 & 0.039 & 0.020 & 0.007 & 0.064 & 0.188 & 0.457 \\
\hline Total NN & AV & 2.984 & 3.091 & 3.060 & 3.018 & 3.001 & 2.997 & 3.002 & 3.020 & 3.082 & 3.207 \\
& STD & 0.000 & 0.150 & 0.132 & 0.058 & 0.029 & 0.017 & 0.022 & 0.058 & 0.141 & 0.289 \\
\hline
\end{tabular}


Table S3. The xyz coordinates of the GB bulk model (before geometry optimization).

\begin{tabular}{|c|c|c|c|c|}
\hline Index & Atom type & $x$ & $\mathrm{y}$ & $\mathrm{z}$ \\
\hline 1 & $\mathrm{Au}$ & 1.49219 & 0.00000 & 0.00000 \\
\hline 2 & $\mathrm{Au}$ & 4.47615 & 0.00000 & 0.00000 \\
\hline 3 & $\mathrm{Au}$ & 7.46012 & 0.00000 & 0.00000 \\
\hline 4 & $\mathrm{Au}$ & 0.00000 & 2.23365 & 0.00000 \\
\hline 5 & $\mathrm{Au}$ & 2.98396 & 2.23365 & 0.00000 \\
\hline 6 & $\mathrm{Au}$ & 5.96792 & 2.23365 & 0.00000 \\
\hline 7 & $\mathrm{Au}$ & 1.49219 & 4.81797 & 0.00000 \\
\hline 8 & $\mathrm{Au}$ & 4.47615 & 4.81797 & 0.00000 \\
\hline 9 & $\mathrm{Au}$ & 7.46012 & 4.81797 & 0.00000 \\
\hline 10 & $\mathrm{Au}$ & 2.98396 & 7.40188 & 0.00000 \\
\hline 11 & $\mathrm{Au}$ & 0.00000 & 7.40188 & 0.00000 \\
\hline 12 & $\mathrm{Au}$ & 5.96792 & 7.40188 & 0.00000 \\
\hline 13 & $\mathrm{Au}$ & 1.49219 & 9.98621 & 0.00000 \\
\hline 14 & $\mathrm{Au}$ & 4.47615 & 9.98621 & 0.00000 \\
\hline 15 & $\mathrm{Au}$ & 7.46012 & 9.98621 & 0.00000 \\
\hline 16 & $\mathrm{Au}$ & 5.96792 & 12.57054 & 0.00000 \\
\hline 17 & $\mathrm{Au}$ & 0.00000 & 12.57054 & 0.00000 \\
\hline 18 & $\mathrm{Au}$ & 2.98396 & 12.57054 & 0.00000 \\
\hline 19 & $\mathrm{Au}$ & 4.47615 & 15.15486 & 0.00000 \\
\hline 20 & $\mathrm{Au}$ & 7.46012 & 15.15486 & 0.00000 \\
\hline 21 & $\mathrm{Au}$ & 1.49219 & 15.15486 & 0.00000 \\
\hline 22 & $\mathrm{Au}$ & 0.00000 & 17.73877 & 0.00000 \\
\hline 23 & $\mathrm{Au}$ & 2.98396 & 17.73877 & 0.00000 \\
\hline 24 & $\mathrm{Au}$ & 5.96792 & 17.73877 & 0.00000 \\
\hline 25 & $\mathrm{Au}$ & 1.49219 & 20.32310 & 0.00000 \\
\hline 26 & $\mathrm{Au}$ & 4.47615 & 20.32310 & 0.00000 \\
\hline 27 & $\mathrm{Au}$ & 7.46012 & 20.32310 & 0.00000 \\
\hline 28 & $\mathrm{Au}$ & 1.49219 & 23.06694 & 0.00000 \\
\hline 29 & $\mathrm{Au}$ & 4.47615 & 23.06694 & 0.00000 \\
\hline 30 & $\mathrm{Au}$ & 7.46012 & 23.06694 & 0.00000 \\
\hline 31 & $\mathrm{Au}$ & 0.00000 & 25.65127 & 0.00000 \\
\hline 32 & $\mathrm{Au}$ & 2.98396 & 25.65127 & 0.00000 \\
\hline 33 & $\mathrm{Au}$ & 5.96792 & 25.65127 & 0.00000 \\
\hline 34 & $\mathrm{Au}$ & 4.47615 & 28.23560 & 0.00000 \\
\hline 35 & $\mathrm{Au}$ & 7.46012 & 28.23560 & 0.00000 \\
\hline 36 & $\mathrm{Au}$ & 1.49219 & 28.23560 & 0.00000 \\
\hline 37 & $\mathrm{Au}$ & 5.96792 & 30.81993 & 0.00000 \\
\hline 38 & $\mathrm{Au}$ & 0.00000 & 30.81993 & 0.00000 \\
\hline 39 & $\mathrm{Au}$ & 2.98396 & 30.81993 & 0.00000 \\
\hline 40 & $\mathrm{Au}$ & 1.49219 & 33.40383 & 0.00000 \\
\hline 41 & $\mathrm{Au}$ & 4.47615 & 33.40383 & 0.00000 \\
\hline 42 & $\mathrm{Au}$ & 7.46012 & 33.40383 & 0.00000 \\
\hline 43 & $\mathrm{Au}$ & 2.98396 & 35.98816 & 0.00000 \\
\hline 44 & $\mathrm{Au}$ & 0.00000 & 35.98816 & 0.00000 \\
\hline 45 & $\mathrm{Au}$ & 5.96792 & 35.98816 & 0.00000 \\
\hline 46 & $\mathrm{Au}$ & 1.49219 & 38.57249 & 0.00000 \\
\hline 47 & $\mathrm{Au}$ & 4.47615 & 38.57249 & 0.00000 \\
\hline 48 & $\mathrm{Au}$ & 7.46012 & 38.57249 & 0.00000 \\
\hline 49 & $\mathrm{Au}$ & 0.00000 & 41.15682 & 0.00000 \\
\hline
\end{tabular}




\begin{tabular}{|c|c|c|c|c|}
\hline 50 & $\mathrm{Au}$ & 2.98396 & 41.15682 & 0.00000 \\
\hline 51 & $\mathrm{Au}$ & 5.96792 & 41.15682 & 0.00000 \\
\hline 52 & $\mathrm{Au}$ & 1.49219 & 1.37234 & 2.43663 \\
\hline 53 & $\mathrm{Au}$ & 4.47615 & 1.37234 & 2.43663 \\
\hline 54 & $\mathrm{Au}$ & 7.46012 & 1.37234 & 2.43663 \\
\hline 55 & $\mathrm{Au}$ & 0.00000 & 3.95625 & 2.43663 \\
\hline 56 & $\mathrm{Au}$ & 2.98396 & 3.95625 & 2.43663 \\
\hline 57 & $\mathrm{Au}$ & 5.96792 & 3.95625 & 2.43663 \\
\hline 58 & $\mathrm{Au}$ & 1.49219 & 6.54058 & 2.43663 \\
\hline 59 & $\mathrm{Au}$ & 4.47615 & 6.54058 & 2.43663 \\
\hline 60 & $\mathrm{Au}$ & 7.46012 & 6.54058 & 2.43663 \\
\hline 61 & $\mathrm{Au}$ & 5.96792 & 9.12491 & 2.43663 \\
\hline 62 & $\mathrm{Au}$ & 0.00000 & 9.12491 & 2.43663 \\
\hline 63 & $\mathrm{Au}$ & 2.98396 & 9.12491 & 2.43663 \\
\hline 64 & $\mathrm{Au}$ & 4.47615 & 11.70923 & 2.43663 \\
\hline 65 & $\mathrm{Au}$ & 7.46012 & 11.70923 & 2.43663 \\
\hline 66 & $\mathrm{Au}$ & 1.49219 & 11.70923 & 2.43663 \\
\hline 67 & $\mathrm{Au}$ & 0.00000 & 14.29314 & 2.43663 \\
\hline 68 & $\mathrm{Au}$ & 2.98396 & 14.29314 & 2.43663 \\
\hline 69 & $\mathrm{Au}$ & 5.96792 & 14.29314 & 2.43663 \\
\hline 70 & $\mathrm{Au}$ & 1.49219 & 16.87747 & 2.43663 \\
\hline 71 & $\mathrm{Au}$ & 4.47615 & 16.87747 & 2.43663 \\
\hline 72 & $\mathrm{Au}$ & 7.46012 & 16.87747 & 2.43663 \\
\hline 73 & $\mathrm{Au}$ & 0.00000 & 19.46180 & 2.43663 \\
\hline 74 & $\mathrm{Au}$ & 2.98396 & 19.46180 & 2.43663 \\
\hline 75 & $\mathrm{Au}$ & 5.96792 & 19.46180 & 2.43663 \\
\hline 76 & $\mathrm{Au}$ & 1.49219 & 21.69502 & 2.43663 \\
\hline 77 & $\mathrm{Au}$ & 4.47615 & 21.69502 & 2.43663 \\
\hline 78 & $\mathrm{Au}$ & 7.46012 & 21.69502 & 2.43663 \\
\hline 79 & $\mathrm{Au}$ & 0.00000 & 23.92867 & 2.43663 \\
\hline 80 & $\mathrm{Au}$ & 2.98396 & 23.92867 & 2.43663 \\
\hline 81 & $\mathrm{Au}$ & 5.96792 & 23.92867 & 2.43663 \\
\hline 82 & $\mathrm{Au}$ & 1.49219 & 26.51257 & 2.43663 \\
\hline 83 & $\mathrm{Au}$ & 4.47615 & 26.51257 & 2.43663 \\
\hline 84 & $\mathrm{Au}$ & 7.46012 & 26.51257 & 2.43663 \\
\hline 85 & $\mathrm{Au}$ & 0.00000 & 29.09690 & 2.43663 \\
\hline 86 & $\mathrm{Au}$ & 2.98396 & 29.09690 & 2.43663 \\
\hline 87 & $\mathrm{Au}$ & 5.96792 & 29.09690 & 2.43663 \\
\hline 88 & $\mathrm{Au}$ & 4.47615 & 31.68123 & 2.43663 \\
\hline 89 & $\mathrm{Au}$ & 7.46012 & 31.68123 & 2.43663 \\
\hline 90 & $\mathrm{Au}$ & 1.49219 & 31.68123 & 2.43663 \\
\hline 91 & $\mathrm{Au}$ & 5.96792 & 34.26556 & 2.43663 \\
\hline 92 & $\mathrm{Au}$ & 0.00000 & 34.26556 & 2.43663 \\
\hline 93 & $\mathrm{Au}$ & 2.98396 & 34.26556 & 2.43663 \\
\hline 94 & $\mathrm{Au}$ & 1.49219 & 36.84946 & 2.43663 \\
\hline 95 & $\mathrm{Au}$ & 4.47615 & 36.84946 & 2.43663 \\
\hline 96 & $\mathrm{Au}$ & 7.46012 & 36.84946 & 2.43663 \\
\hline 97 & $\mathrm{Au}$ & 0.00000 & 39.43379 & 2.43663 \\
\hline 98 & $\mathrm{Au}$ & 2.98396 & 39.43379 & 2.43663 \\
\hline 99 & $\mathrm{Au}$ & 5.96792 & 39.43379 & 2.43663 \\
\hline 100 & $\mathrm{Au}$ & 1.49219 & 42.01812 & 2.43663 \\
\hline 101 & $\mathrm{Au}$ & 4.47615 & 42.01812 & 2.43663 \\
\hline
\end{tabular}




\begin{tabular}{|c|c|c|c|c|}
\hline 102 & $\mathrm{Au}$ & 7.46012 & 42.01812 & 2.43663 \\
\hline 103 & $\mathrm{Au}$ & 0.00000 & 0.00000 & 4.87283 \\
\hline 104 & $\mathrm{Au}$ & 2.98396 & 0.00000 & 4.87283 \\
\hline 105 & $\mathrm{Au}$ & 5.96792 & 0.00000 & 4.87283 \\
\hline 106 & $\mathrm{Au}$ & 1.49219 & 3.09495 & 4.87283 \\
\hline 107 & $\mathrm{Au}$ & 4.47615 & 3.09495 & 4.87283 \\
\hline 108 & $\mathrm{Au}$ & 7.46012 & 3.09495 & 4.87283 \\
\hline 109 & $\mathrm{Au}$ & 0.00000 & 5.67928 & 4.87283 \\
\hline 110 & $\mathrm{Au}$ & 2.98396 & 5.67928 & 4.87283 \\
\hline 111 & $\overline{\mathrm{Au}}$ & 5.96792 & 5.67928 & 4.87283 \\
\hline 112 & $\mathrm{Au}$ & 1.49219 & 8.26360 & 4.87283 \\
\hline 113 & $\mathrm{Au}$ & 4.47615 & 8.26360 & 4.87283 \\
\hline 114 & $\mathrm{Au}$ & 7.46012 & 8.26360 & 4.87283 \\
\hline 115 & $\mathrm{Au}$ & 0.00000 & 10.84751 & 4.87283 \\
\hline 116 & $\mathrm{Au}$ & 2.98396 & 10.84751 & 4.87283 \\
\hline 117 & $\mathrm{Au}$ & 5.96792 & 10.84751 & 4.87283 \\
\hline 118 & $\mathrm{Au}$ & 1.49219 & 13.43184 & 4.87283 \\
\hline 119 & $\mathrm{Au}$ & 4.47615 & 13.43184 & 4.87283 \\
\hline 120 & $\mathrm{Au}$ & 7.46012 & 13.43184 & 4.87283 \\
\hline 121 & $\overline{\mathrm{Au}}$ & 0.00000 & 16.01617 & 4.87283 \\
\hline 122 & $\mathrm{Au}$ & 2.98396 & 16.01617 & 4.87283 \\
\hline 123 & $\mathrm{Au}$ & 5.96792 & 16.01617 & 4.87283 \\
\hline 124 & $\mathrm{Au}$ & 4.47615 & 18.60049 & 4.87283 \\
\hline 125 & $\mathrm{Au}$ & 7.46012 & 18.60049 & 4.87283 \\
\hline 126 & $\mathrm{Au}$ & 1.49219 & 18.60049 & 4.87283 \\
\hline 127 & $\mathrm{Au}$ & 0.00000 & 21.69502 & 4.87283 \\
\hline 128 & $\mathrm{Au}$ & 2.98396 & 21.69502 & 4.87283 \\
\hline 129 & $\mathrm{Au}$ & 5.96792 & 21.69502 & 4.87283 \\
\hline 130 & $\mathrm{Au}$ & 4.47615 & 24.78997 & 4.87283 \\
\hline 131 & $\overline{\mathrm{Au}}$ & 7.46012 & 24.78997 & 4.87283 \\
\hline 132 & $\mathrm{Au}$ & 1.49219 & 24.78997 & 4.87283 \\
\hline 133 & $\mathrm{Au}$ & 0.00000 & 27.37430 & 4.87283 \\
\hline 134 & $\mathrm{Au}$ & 2.98396 & 27.37430 & 4.87283 \\
\hline 135 & $\mathrm{Au}$ & 5.96792 & 27.37430 & 4.87283 \\
\hline 136 & $\mathrm{Au}$ & 1.49219 & 29.95820 & 4.87283 \\
\hline 137 & $\mathrm{Au}$ & 4.47615 & 29.95820 & 4.87283 \\
\hline 138 & $\mathrm{Au}$ & 7.46012 & 29.95820 & 4.87283 \\
\hline 139 & $\mathrm{Au}$ & 0.00000 & 32.54253 & 4.87283 \\
\hline 140 & $\mathrm{Au}$ & 2.98396 & 32.54253 & 4.87283 \\
\hline 141 & $\overline{\mathrm{Au}}$ & 5.96792 & 32.54253 & 4.87283 \\
\hline 142 & $\mathrm{Au}$ & 1.49219 & 35.12686 & 4.87283 \\
\hline 143 & $\mathrm{Au}$ & 4.47615 & 35.12686 & 4.87283 \\
\hline 144 & $\mathrm{Au}$ & 7.46012 & 35.12686 & 4.87283 \\
\hline 145 & $\mathrm{Au}$ & 0.00000 & 37.71119 & 4.87283 \\
\hline 146 & $\mathrm{Au}$ & 2.98396 & 37.71119 & 4.87283 \\
\hline 147 & $\mathrm{Au}$ & 5.96792 & 37.71119 & 4.87283 \\
\hline 148 & $\mathrm{Au}$ & 1.49219 & 40.29509 & 4.87283 \\
\hline 149 & $\mathrm{Au}$ & 4.47615 & 40.29509 & 4.87283 \\
\hline 150 & $\mathrm{Au}$ & 7.46012 & 40.29509 & 4.87283 \\
\hline
\end{tabular}


Table S4. The xyz coordinates of the GB bulk model (geometry optimized).

\begin{tabular}{|c|c|c|c|c|}
\hline Index & Atom type & $\mathrm{x}$ & $\mathrm{y}$ & $\mathrm{z}$ \\
\hline 1 & $\mathrm{Au}$ & 7.46004 & -0.00005 & -0.31849 \\
\hline 2 & $\mathrm{Au}$ & 1.49206 & -0.00005 & -0.31849 \\
\hline 3 & $\mathrm{Au}$ & 4.47605 & -0.00004 & -0.31849 \\
\hline 4 & $\mathrm{Au}$ & 2.98405 & 2.41668 & -0.01773 \\
\hline 5 & $\mathrm{Au}$ & 5.96805 & 2.41668 & -0.01774 \\
\hline 6 & $\mathrm{Au}$ & 0.00006 & 2.41668 & -0.01775 \\
\hline 7 & $\mathrm{Au}$ & 4.47604 & 4.89587 & 0.05231 \\
\hline 8 & $\mathrm{Au}$ & 1.49205 & 4.89587 & 0.05231 \\
\hline 9 & $\mathrm{Au}$ & 7.46003 & 4.89587 & 0.05232 \\
\hline 10 & $\mathrm{Au}$ & 5.96802 & 7.42686 & 0.04496 \\
\hline 11 & $\mathrm{Au}$ & 0.00003 & 7.42686 & 0.04494 \\
\hline 12 & $\mathrm{Au}$ & 2.98403 & 7.42686 & 0.04497 \\
\hline 13 & $\mathrm{Au}$ & 4.47602 & 9.99048 & 0.01058 \\
\hline 14 & $\mathrm{Au}$ & 7.46001 & 9.99049 & 0.01057 \\
\hline 15 & $\mathrm{Au}$ & 1.49203 & 9.99049 & 0.01057 \\
\hline 16 & $\mathrm{Au}$ & 2.98402 & 12.56529 & -0.03519 \\
\hline 17 & $\mathrm{Au}$ & 5.96800 & 12.56529 & -0.03519 \\
\hline 18 & $\mathrm{Au}$ & 0.00002 & 12.56530 & -0.03521 \\
\hline 19 & $\mathrm{Au}$ & 4.47603 & 15.13962 & -0.07584 \\
\hline 20 & $\mathrm{Au}$ & 1.49204 & 15.13963 & -0.07585 \\
\hline 21 & $\mathrm{Au}$ & 7.46001 & 15.13964 & -0.07584 \\
\hline 22 & $\mathrm{Au}$ & 2.98405 & 17.70808 & -0.07951 \\
\hline 23 & $\mathrm{Au}$ & 0.00005 & 17.70809 & -0.07952 \\
\hline 24 & $\mathrm{Au}$ & 5.96803 & 17.70809 & -0.07951 \\
\hline 25 & $\mathrm{Au}$ & 1.49206 & 20.27644 & 0.09499 \\
\hline 26 & $\mathrm{Au}$ & 4.47605 & 20.27645 & 0.09499 \\
\hline 27 & $\mathrm{Au}$ & 7.46004 & 20.27645 & 0.09500 \\
\hline 28 & $\mathrm{Au}$ & 1.49205 & 23.11457 & 0.09511 \\
\hline 29 & $\mathrm{Au}$ & 7.46004 & 23.11457 & 0.09511 \\
\hline 30 & $\mathrm{Au}$ & 4.47604 & 23.11457 & 0.09511 \\
\hline 31 & $\mathrm{Au}$ & 2.98404 & 25.68249 & -0.07922 \\
\hline 32 & $\mathrm{Au}$ & 5.96803 & 25.68250 & -0.07922 \\
\hline 33 & $\mathrm{Au}$ & 0.00005 & 25.68251 & -0.07921 \\
\hline 34 & $\mathrm{Au}$ & 4.47604 & 28.25059 & -0.07580 \\
\hline 35 & $\mathrm{Au}$ & 1.49205 & 28.25060 & -0.07580 \\
\hline 36 & $\mathrm{Au}$ & 7.46002 & 28.25060 & -0.07581 \\
\hline 37 & $\mathrm{Au}$ & 2.98403 & 30.82469 & -0.03532 \\
\hline 38 & $\mathrm{Au}$ & 5.96802 & 30.82470 & -0.03531 \\
\hline 39 & $\mathrm{Au}$ & 0.00004 & 30.82470 & -0.03531 \\
\hline 40 & $\mathrm{Au}$ & 4.47602 & 33.39928 & 0.01034 \\
\hline 41 & $\mathrm{Au}$ & 1.49203 & 33.39929 & 0.01035 \\
\hline 42 & $\mathrm{Au}$ & 7.46001 & 33.39930 & 0.01034 \\
\hline 43 & $\mathrm{Au}$ & 2.98402 & 35.96253 & 0.04472 \\
\hline 44 & $\mathrm{Au}$ & 5.96800 & 35.96254 & 0.04472 \\
\hline 45 & $\mathrm{Au}$ & 0.00002 & 35.96255 & 0.04473 \\
\hline 46 & $\mathrm{Au}$ & 4.47603 & 38.49360 & 0.05208 \\
\hline 47 & $\mathrm{Au}$ & 1.49204 & 38.49361 & 0.05208 \\
\hline 48 & $\mathrm{Au}$ & 7.46002 & 38.49362 & 0.05208 \\
\hline 49 & $\mathrm{Au}$ & 2.98405 & 40.97247 & -0.01816 \\
\hline
\end{tabular}




\begin{tabular}{|c|c|c|c|c|}
\hline 50 & $\mathrm{Au}$ & 5.96803 & 40.97247 & -0.01815 \\
\hline 51 & $\mathrm{Au}$ & 0.00006 & 40.97247 & -0.01816 \\
\hline 52 & $\mathrm{Au}$ & 7.46005 & 1.41867 & 2.34148 \\
\hline 53 & $\mathrm{Au}$ & 4.47606 & 1.41867 & 2.34148 \\
\hline 54 & $\mathrm{Au}$ & 1.49206 & 1.41867 & 2.34147 \\
\hline 55 & $\mathrm{Au}$ & 5.96804 & 3.98634 & 2.51588 \\
\hline 56 & $\mathrm{Au}$ & 2.98405 & 3.98634 & 2.51588 \\
\hline 57 & $\mathrm{Au}$ & 0.00006 & 3.98634 & 2.51586 \\
\hline 58 & $\mathrm{Au}$ & 1.49203 & 6.55440 & 2.51226 \\
\hline 59 & $\mathrm{Au}$ & 4.47603 & 6.55440 & 2.51227 \\
\hline 60 & $\mathrm{Au}$ & 7.46002 & 6.55440 & 2.51226 \\
\hline 61 & $\mathrm{Au}$ & 5.96802 & 9.12879 & 2.47163 \\
\hline 62 & $\mathrm{Au}$ & 0.00004 & 9.12880 & 2.47161 \\
\hline 63 & $\mathrm{Au}$ & 2.98402 & 9.12880 & 2.47164 \\
\hline 64 & $\mathrm{Au}$ & 4.47602 & 11.70364 & 2.42585 \\
\hline 65 & $\mathrm{Au}$ & 7.46001 & 11.70365 & 2.42584 \\
\hline 66 & $\mathrm{Au}$ & 1.49203 & 11.70365 & 2.42584 \\
\hline 67 & $\mathrm{Au}$ & 2.98403 & 14.26712 & 2.39152 \\
\hline 68 & $\mathrm{Au}$ & 5.96802 & 14.26712 & 2.39153 \\
\hline 69 & $\mathrm{Au}$ & 0.00004 & 14.26712 & 2.39151 \\
\hline 70 & $\mathrm{Au}$ & 4.47603 & 16.79842 & 2.38424 \\
\hline 71 & $\mathrm{Au}$ & 1.49204 & 16.79842 & 2.38424 \\
\hline 72 & $\mathrm{Au}$ & 7.46003 & 16.79843 & 2.38424 \\
\hline 73 & $\mathrm{Au}$ & 2.98405 & 19.27787 & 2.45432 \\
\hline 74 & $\mathrm{Au}$ & 0.00006 & 19.27787 & 2.45431 \\
\hline 75 & $\mathrm{Au}$ & 5.96803 & 19.27788 & 2.45432 \\
\hline 76 & $\mathrm{Au}$ & 4.47605 & 21.69547 & 2.75504 \\
\hline 77 & $\mathrm{Au}$ & 1.49205 & 21.69547 & 2.75504 \\
\hline 78 & $\mathrm{Au}$ & 7.46003 & 21.69548 & 2.75504 \\
\hline 79 & $\mathrm{Au}$ & 2.98405 & 24.11242 & 2.45455 \\
\hline 80 & $\mathrm{Au}$ & 5.96804 & 24.11242 & 2.45455 \\
\hline 81 & $\mathrm{Au}$ & 0.00006 & 24.11243 & 2.45455 \\
\hline 82 & $\mathrm{Au}$ & 4.47604 & 26.59183 & 2.38433 \\
\hline 83 & $\mathrm{Au}$ & 7.46003 & 26.59184 & 2.38431 \\
\hline 84 & $\mathrm{Au}$ & 1.49205 & 26.59184 & 2.38433 \\
\hline 85 & $\mathrm{Au}$ & 5.96803 & 29.12284 & 2.39142 \\
\hline 86 & $\mathrm{Au}$ & 2.98405 & 29.12285 & 2.39143 \\
\hline 87 & $\mathrm{Au}$ & 0.00005 & 29.12285 & 2.39143 \\
\hline 88 & $\mathrm{Au}$ & 7.46002 & 31.68643 & 2.42573 \\
\hline 89 & $\mathrm{Au}$ & 4.47603 & 31.68643 & 2.42574 \\
\hline 90 & $\mathrm{Au}$ & 1.49205 & 31.68643 & 2.42574 \\
\hline 91 & $\mathrm{Au}$ & 2.98403 & 34.26099 & 2.47153 \\
\hline 92 & $\mathrm{Au}$ & 0.00003 & 34.26099 & 2.47152 \\
\hline 93 & $\mathrm{Au}$ & 5.96802 & 34.26099 & 2.47152 \\
\hline 94 & $\mathrm{Au}$ & 4.47602 & 36.83504 & 2.51213 \\
\hline 95 & $\mathrm{Au}$ & 1.49203 & 36.83504 & 2.51212 \\
\hline 96 & $\mathrm{Au}$ & 7.46001 & 36.83504 & 2.51212 \\
\hline 97 & $\mathrm{Au}$ & 5.96802 & 39.40300 & 2.51544 \\
\hline 98 & $\mathrm{Au}$ & 0.00004 & 39.40300 & 2.51544 \\
\hline 99 & $\mathrm{Au}$ & 2.98403 & 39.40300 & 2.51544 \\
\hline 100 & $\mathrm{Au}$ & 4.47605 & 41.97065 & 2.34100 \\
\hline 101 & $\mathrm{Au}$ & 7.46004 & 41.97065 & 2.34099 \\
\hline
\end{tabular}




\begin{tabular}{|c|c|c|c|c|}
\hline 102 & $\mathrm{Au}$ & 1.49206 & 41.97066 & 2.34099 \\
\hline 103 & $\mathrm{Au}$ & 0.00007 & -0.00068 & 4.48238 \\
\hline 104 & $\mathrm{Au}$ & 5.96806 & -0.00068 & 4.48238 \\
\hline 105 & $\mathrm{Au}$ & 2.98406 & -0.00067 & 4.48238 \\
\hline 106 & $\mathrm{Au}$ & 4.47606 & 2.87463 & 4.84946 \\
\hline 107 & $\mathrm{Au}$ & 1.49207 & 2.87463 & 4.84945 \\
\hline 108 & $\mathrm{Au}$ & 7.46005 & 2.87465 & 4.84944 \\
\hline 109 & $\mathrm{Au}$ & 0.00004 & 5.59688 & 4.93386 \\
\hline 110 & $\mathrm{Au}$ & 2.98404 & 5.59689 & 4.93387 \\
\hline 111 & $\overline{\mathrm{Au}}$ & 5.96802 & 5.59689 & 4.93386 \\
\hline 112 & $\mathrm{Au}$ & 1.49204 & 8.23677 & 4.91437 \\
\hline 113 & $\mathrm{Au}$ & 7.46002 & 8.23678 & 4.91437 \\
\hline 114 & $\mathrm{Au}$ & 4.47602 & 8.23678 & 4.91438 \\
\hline 115 & $\mathrm{Au}$ & 5.96801 & 10.84695 & 4.87278 \\
\hline 116 & $\mathrm{Au}$ & 0.00003 & 10.84695 & 4.87277 \\
\hline 117 & $\mathrm{Au}$ & 2.98403 & 10.84695 & 4.87278 \\
\hline 118 & $\mathrm{Au}$ & 1.49203 & 13.45711 & 4.83130 \\
\hline 119 & $\mathrm{Au}$ & 4.47602 & 13.45712 & 4.83131 \\
\hline 120 & $\mathrm{Au}$ & 7.46001 & 13.45712 & 4.83131 \\
\hline 121 & $\overline{\mathrm{Au}}$ & 0.00002 & 16.09706 & 4.81187 \\
\hline 122 & $\mathrm{Au}$ & 2.98403 & 16.09706 & 4.81187 \\
\hline 123 & $\mathrm{Au}$ & 5.96801 & 16.09707 & 4.81187 \\
\hline 124 & $\mathrm{Au}$ & 1.49205 & 18.81968 & 4.89659 \\
\hline 125 & $\mathrm{Au}$ & 4.47604 & 18.81969 & 4.89660 \\
\hline 126 & $\mathrm{Au}$ & 7.46003 & 18.81970 & 4.89659 \\
\hline 127 & $\mathrm{Au}$ & 0.00006 & 21.69589 & 5.26371 \\
\hline 128 & $\mathrm{Au}$ & 2.98405 & 21.69589 & 5.26370 \\
\hline 129 & $\mathrm{Au}$ & 5.96804 & 21.69590 & 5.26370 \\
\hline 130 & $\mathrm{Au}$ & 4.47605 & 24.57122 & 4.89663 \\
\hline 131 & $\overline{\mathrm{Au}}$ & 7.46004 & 24.57123 & 4.89663 \\
\hline 132 & $\mathrm{Au}$ & 1.49205 & 24.57123 & 4.89663 \\
\hline 133 & $\mathrm{Au}$ & 5.96804 & 27.29319 & 4.81189 \\
\hline 134 & $\mathrm{Au}$ & 2.98405 & 27.29320 & 4.81190 \\
\hline 135 & $\mathrm{Au}$ & 0.00005 & 27.29320 & 4.81190 \\
\hline 136 & $\mathrm{Au}$ & 4.47604 & 29.93285 & 4.83121 \\
\hline 137 & $\mathrm{Au}$ & 1.49204 & 29.93286 & 4.83121 \\
\hline 138 & $\mathrm{Au}$ & 7.46003 & 29.93287 & 4.83120 \\
\hline 139 & $\mathrm{Au}$ & 2.98404 & 32.54297 & 4.87262 \\
\hline 140 & $\mathrm{Au}$ & 5.96802 & 32.54297 & 4.87262 \\
\hline 141 & $\overline{\mathrm{Au}}$ & 0.00005 & 32.54298 & 4.87262 \\
\hline 142 & $\mathrm{Au}$ & 4.47602 & 35.15295 & 4.91425 \\
\hline 143 & $\mathrm{Au}$ & 1.49202 & 35.15295 & 4.91426 \\
\hline 144 & $\mathrm{Au}$ & 7.46000 & 35.15296 & 4.91426 \\
\hline 145 & $\mathrm{Au}$ & 5.96801 & 37.79263 & 4.93366 \\
\hline 146 & $\mathrm{Au}$ & 2.98402 & 37.79263 & 4.93366 \\
\hline 147 & $\mathrm{Au}$ & 0.00003 & 37.79264 & 4.93367 \\
\hline 148 & $\mathrm{Au}$ & 7.46003 & 40.51480 & 4.84905 \\
\hline 149 & $\mathrm{Au}$ & 4.47604 & 40.51480 & 4.84904 \\
\hline 150 & $\mathrm{Au}$ & 1.49205 & 40.51482 & 4.84904 \\
\hline
\end{tabular}


Table S5. The xyz coordinates of the GB slab model (geometry optimized).

\begin{tabular}{|c|c|c|c|c|}
\hline Index & Atom type & $\mathrm{x}$ & $\mathrm{y}$ & $\mathrm{z}$ \\
\hline 1 & $\mathrm{Au}$ & 7.46004 & -0.00005 & -0.31849 \\
\hline 2 & $\mathrm{Au}$ & 1.49206 & -0.00005 & -0.31849 \\
\hline 3 & $\mathrm{Au}$ & 4.47605 & -0.00004 & -0.31849 \\
\hline 4 & $\mathrm{Au}$ & 2.98405 & 2.41668 & -0.01773 \\
\hline 5 & $\mathrm{Au}$ & 5.96805 & 2.41668 & -0.01774 \\
\hline 6 & $\mathrm{Au}$ & 0.00006 & 2.41668 & -0.01775 \\
\hline 7 & $\mathrm{Au}$ & 4.47604 & 4.89587 & 0.05231 \\
\hline 8 & $\mathrm{Au}$ & 1.49205 & 4.89587 & 0.05231 \\
\hline 9 & $\mathrm{Au}$ & 7.46003 & 4.89587 & 0.05232 \\
\hline 10 & $\mathrm{Au}$ & 5.96802 & 7.42686 & 0.04496 \\
\hline 11 & $\mathrm{Au}$ & 0.00003 & 7.42686 & 0.04494 \\
\hline 12 & $\mathrm{Au}$ & 2.98403 & 7.42686 & 0.04497 \\
\hline 13 & $\mathrm{Au}$ & 4.47602 & 9.99048 & 0.01058 \\
\hline 14 & $\mathrm{Au}$ & 7.46001 & 9.99049 & 0.01057 \\
\hline 15 & $\mathrm{Au}$ & 1.49203 & 9.99049 & 0.01057 \\
\hline 16 & $\mathrm{Au}$ & 2.98402 & 12.56529 & -0.03519 \\
\hline 17 & $\mathrm{Au}$ & 5.96800 & 12.56529 & -0.03519 \\
\hline 18 & $\mathrm{Au}$ & 0.00002 & 12.56530 & -0.03521 \\
\hline 19 & $\mathrm{Au}$ & 4.47603 & 15.13962 & -0.07584 \\
\hline 20 & $\mathrm{Au}$ & 1.49204 & 15.13963 & -0.07585 \\
\hline 21 & $\mathrm{Au}$ & 7.46001 & 15.13964 & -0.07584 \\
\hline 22 & $\mathrm{Au}$ & 2.98405 & 17.70808 & -0.07951 \\
\hline 23 & $\mathrm{Au}$ & 0.00005 & 17.70809 & -0.07952 \\
\hline 24 & $\mathrm{Au}$ & 5.96803 & 17.70809 & -0.07951 \\
\hline 25 & $\mathrm{Au}$ & 1.49206 & 20.27644 & 0.09499 \\
\hline 26 & $\mathrm{Au}$ & 4.47605 & 20.27645 & 0.09499 \\
\hline 27 & $\mathrm{Au}$ & 7.46004 & 20.27645 & 0.09500 \\
\hline 28 & $\mathrm{Au}$ & 1.49205 & 23.11457 & 0.09511 \\
\hline 29 & $\mathrm{Au}$ & 7.46004 & 23.11457 & 0.09511 \\
\hline 30 & $\mathrm{Au}$ & 4.47604 & 23.11457 & 0.09511 \\
\hline 31 & $\mathrm{Au}$ & 2.98404 & 25.68249 & -0.07922 \\
\hline 32 & $\mathrm{Au}$ & 5.96803 & 25.68250 & -0.07922 \\
\hline 33 & $\mathrm{Au}$ & 0.00005 & 25.68251 & -0.07921 \\
\hline 34 & $\mathrm{Au}$ & 4.47604 & 28.25059 & -0.07580 \\
\hline 35 & $\mathrm{Au}$ & 1.49205 & 28.25060 & -0.07580 \\
\hline 36 & $\mathrm{Au}$ & 7.46002 & 28.25060 & -0.07581 \\
\hline 37 & $\mathrm{Au}$ & 2.98403 & 30.82469 & -0.03532 \\
\hline 38 & $\mathrm{Au}$ & 5.96802 & 30.82470 & -0.03531 \\
\hline 39 & $\mathrm{Au}$ & 0.00004 & 30.82470 & -0.03531 \\
\hline 40 & $\mathrm{Au}$ & 4.47602 & 33.39928 & 0.01034 \\
\hline 41 & $\mathrm{Au}$ & 1.49203 & 33.39929 & 0.01035 \\
\hline 42 & $\mathrm{Au}$ & 7.46001 & 33.39930 & 0.01034 \\
\hline 43 & $\mathrm{Au}$ & 2.98402 & 35.96253 & 0.04472 \\
\hline 44 & $\mathrm{Au}$ & 5.96800 & 35.96254 & 0.04472 \\
\hline 45 & $\mathrm{Au}$ & 0.00002 & 35.96255 & 0.04473 \\
\hline 46 & $\mathrm{Au}$ & 4.47603 & 38.49360 & 0.05208 \\
\hline 47 & $\mathrm{Au}$ & 1.49204 & 38.49361 & 0.05208 \\
\hline 48 & $\mathrm{Au}$ & 7.46002 & 38.49362 & 0.05208 \\
\hline 49 & $\mathrm{Au}$ & 2.98405 & 40.97247 & -0.01816 \\
\hline
\end{tabular}




\begin{tabular}{|c|c|c|c|c|}
\hline 50 & $\mathrm{Au}$ & 5.96803 & 40.97247 & -0.01815 \\
\hline 51 & $\mathrm{Au}$ & 0.00006 & 40.97247 & -0.01816 \\
\hline 52 & $\mathrm{Au}$ & 7.46005 & 1.41867 & 2.34148 \\
\hline 53 & $\mathrm{Au}$ & 4.47606 & 1.41867 & 2.34148 \\
\hline 54 & $\mathrm{Au}$ & 1.49206 & 1.41867 & 2.34147 \\
\hline 55 & $\mathrm{Au}$ & 5.96804 & 3.98634 & 2.51588 \\
\hline 56 & $\mathrm{Au}$ & 2.98405 & 3.98634 & 2.51588 \\
\hline 57 & $\mathrm{Au}$ & 0.00006 & 3.98634 & 2.51586 \\
\hline 58 & $\mathrm{Au}$ & 1.49203 & 6.55440 & 2.51226 \\
\hline 59 & $\mathrm{Au}$ & 4.47603 & 6.55440 & 2.51227 \\
\hline 60 & $\mathrm{Au}$ & 7.46002 & 6.55440 & 2.51226 \\
\hline 61 & $\mathrm{Au}$ & 5.96802 & 9.12879 & 2.47163 \\
\hline 62 & $\mathrm{Au}$ & 0.00004 & 9.12880 & 2.47161 \\
\hline 63 & $\mathrm{Au}$ & 2.98402 & 9.12880 & 2.47164 \\
\hline 64 & $\mathrm{Au}$ & 4.47602 & 11.70364 & 2.42585 \\
\hline 65 & $\mathrm{Au}$ & 7.46001 & 11.70365 & 2.42584 \\
\hline 66 & $\mathrm{Au}$ & 1.49203 & 11.70365 & 2.42584 \\
\hline 67 & $\mathrm{Au}$ & 2.98403 & 14.26712 & 2.39152 \\
\hline 68 & $\mathrm{Au}$ & 5.96802 & 14.26712 & 2.39153 \\
\hline 69 & $\mathrm{Au}$ & 0.00004 & 14.26712 & 2.39151 \\
\hline 70 & $\mathrm{Au}$ & 4.47603 & 16.79842 & 2.38424 \\
\hline 71 & $\mathrm{Au}$ & 1.49204 & 16.79842 & 2.38424 \\
\hline 72 & $\mathrm{Au}$ & 7.46003 & 16.79843 & 2.38424 \\
\hline 73 & $\mathrm{Au}$ & 2.98405 & 19.27787 & 2.45432 \\
\hline 74 & $\mathrm{Au}$ & 0.00006 & 19.27787 & 2.45431 \\
\hline 75 & $\mathrm{Au}$ & 5.96803 & 19.27788 & 2.45432 \\
\hline 76 & $\mathrm{Au}$ & 4.47605 & 21.69547 & 2.75504 \\
\hline 77 & $\mathrm{Au}$ & 1.49205 & 21.69547 & 2.75504 \\
\hline 78 & $\mathrm{Au}$ & 7.46003 & 21.69548 & 2.75504 \\
\hline 79 & $\mathrm{Au}$ & 2.98405 & 24.11242 & 2.45455 \\
\hline 80 & $\mathrm{Au}$ & 5.96804 & 24.11242 & 2.45455 \\
\hline 81 & $\mathrm{Au}$ & 0.00006 & 24.11243 & 2.45455 \\
\hline 82 & $\mathrm{Au}$ & 4.47604 & 26.59183 & 2.38433 \\
\hline 83 & $\mathrm{Au}$ & 7.46003 & 26.59184 & 2.38431 \\
\hline 84 & $\mathrm{Au}$ & 1.49205 & 26.59184 & 2.38433 \\
\hline 85 & $\mathrm{Au}$ & 5.96803 & 29.12284 & 2.39142 \\
\hline 86 & $\mathrm{Au}$ & 2.98405 & 29.12285 & 2.39143 \\
\hline 87 & $\mathrm{Au}$ & 0.00005 & 29.12285 & 2.39143 \\
\hline 88 & $\mathrm{Au}$ & 7.46002 & 31.68643 & 2.42573 \\
\hline 89 & $\mathrm{Au}$ & 4.47603 & 31.68643 & 2.42574 \\
\hline 90 & $\mathrm{Au}$ & 1.49205 & 31.68643 & 2.42574 \\
\hline 91 & $\mathrm{Au}$ & 2.98403 & 34.26099 & 2.47153 \\
\hline 92 & $\mathrm{Au}$ & 0.00003 & 34.26099 & 2.47152 \\
\hline 93 & $\mathrm{Au}$ & 5.96802 & 34.26099 & 2.47152 \\
\hline 94 & $\mathrm{Au}$ & 4.47602 & 36.83504 & 2.51213 \\
\hline 95 & $\mathrm{Au}$ & 1.49203 & 36.83504 & 2.51212 \\
\hline 96 & $\mathrm{Au}$ & 7.46001 & 36.83504 & 2.51212 \\
\hline 97 & $\mathrm{Au}$ & 5.96802 & 39.40300 & 2.51544 \\
\hline 98 & $\mathrm{Au}$ & 0.00004 & 39.40300 & 2.51544 \\
\hline 99 & $\mathrm{Au}$ & 2.98403 & 39.40300 & 2.51544 \\
\hline 100 & $\mathrm{Au}$ & 4.47605 & 41.97065 & 2.34100 \\
\hline 101 & $\mathrm{Au}$ & 7.46004 & 41.97065 & 2.34099 \\
\hline
\end{tabular}




\begin{tabular}{|c|c|c|c|c|}
\hline 102 & $\mathrm{Au}$ & 1.49206 & 41.97066 & 2.34099 \\
\hline 103 & $\mathrm{Au}$ & 0.00008 & -0.00071 & 4.48287 \\
\hline 104 & $\mathrm{Au}$ & 5.96805 & -0.00071 & 4.48287 \\
\hline 105 & $\mathrm{Au}$ & 2.98406 & -0.00070 & 4.48287 \\
\hline 106 & $\mathrm{Au}$ & 4.47605 & 2.88242 & 4.84205 \\
\hline 107 & $\mathrm{Au}$ & 1.49206 & 2.88242 & 4.84205 \\
\hline 108 & $\mathrm{Au}$ & 7.46005 & 2.88243 & 4.84205 \\
\hline 109 & $\mathrm{Au}$ & 0.00005 & 5.59364 & 4.94306 \\
\hline 110 & $\mathrm{Au}$ & 5.96802 & 5.59365 & 4.94306 \\
\hline 111 & $\overline{\mathrm{Au}}$ & 2.98403 & 5.59366 & 4.94307 \\
\hline 112 & $\mathrm{Au}$ & 1.49203 & 8.23518 & 4.92372 \\
\hline 113 & $\mathrm{Au}$ & 7.46002 & 8.23519 & 4.92372 \\
\hline 114 & $\mathrm{Au}$ & 4.47602 & 8.23519 & 4.92374 \\
\hline 115 & $\mathrm{Au}$ & 0.00004 & 10.85081 & 4.88307 \\
\hline 116 & $\mathrm{Au}$ & 5.96801 & 10.85081 & 4.88307 \\
\hline 117 & $\mathrm{Au}$ & 2.98403 & 10.85081 & 4.88308 \\
\hline 118 & $\mathrm{Au}$ & 7.46001 & 13.46706 & 4.84478 \\
\hline 119 & $\mathrm{Au}$ & 1.49203 & 13.46706 & 4.84478 \\
\hline 120 & $\mathrm{Au}$ & 4.47602 & 13.46706 & 4.84478 \\
\hline 121 & $\overline{\mathrm{Au}}$ & 0.00005 & 16.11027 & 4.83084 \\
\hline 122 & $\mathrm{Au}$ & 5.96802 & 16.11027 & 4.83084 \\
\hline 123 & $\mathrm{Au}$ & 2.98403 & 16.11028 & 4.83084 \\
\hline 124 & $\mathrm{Au}$ & 1.49205 & 18.82111 & 4.91676 \\
\hline 125 & $\mathrm{Au}$ & 4.47604 & 18.82111 & 4.91675 \\
\hline 126 & $\mathrm{Au}$ & 7.46003 & 18.82113 & 4.91676 \\
\hline 127 & $\mathrm{Au}$ & 0.00006 & 21.69591 & 5.21564 \\
\hline 128 & $\mathrm{Au}$ & 2.98405 & 21.69591 & 5.21566 \\
\hline 129 & $\mathrm{Au}$ & 5.96804 & 21.69592 & 5.21566 \\
\hline 130 & $\mathrm{Au}$ & 7.46003 & 24.56970 & 4.91708 \\
\hline 131 & $\overline{\mathrm{Au}}$ & 4.47604 & 24.56970 & 4.91707 \\
\hline 132 & $\mathrm{Au}$ & 1.49205 & 24.56971 & 4.91708 \\
\hline 133 & $\mathrm{Au}$ & 2.98405 & 27.28003 & 4.83110 \\
\hline 134 & $\mathrm{Au}$ & 5.96804 & 27.28003 & 4.83109 \\
\hline 135 & $\mathrm{Au}$ & 0.00006 & 27.28004 & 4.83110 \\
\hline 136 & $\mathrm{Au}$ & 4.47604 & 29.92301 & 4.84478 \\
\hline 137 & $\mathrm{Au}$ & 1.49205 & 29.92302 & 4.84479 \\
\hline 138 & $\mathrm{Au}$ & 7.46003 & 29.92302 & 4.84479 \\
\hline 139 & $\mathrm{Au}$ & 2.98404 & 32.53908 & 4.88322 \\
\hline 140 & $\mathrm{Au}$ & 5.96802 & 32.53909 & 4.88322 \\
\hline 141 & $\overline{\mathrm{Au}}$ & 0.00005 & 32.53910 & 4.88322 \\
\hline 142 & $\mathrm{Au}$ & 4.47602 & 35.15455 & 4.92397 \\
\hline 143 & $\mathrm{Au}$ & 7.46001 & 35.15455 & 4.92397 \\
\hline 144 & $\mathrm{Au}$ & 1.49203 & 35.15456 & 4.92398 \\
\hline 145 & $\mathrm{Au}$ & 2.98402 & 37.79599 & 4.94302 \\
\hline 146 & $\mathrm{Au}$ & 0.00004 & 37.79599 & 4.94302 \\
\hline 147 & $\mathrm{Au}$ & 5.96801 & 37.79600 & 4.94302 \\
\hline 148 & $\mathrm{Au}$ & 4.47604 & 40.50703 & 4.84179 \\
\hline 149 & $\mathrm{Au}$ & 7.46002 & 40.50704 & 4.84179 \\
\hline 150 & $\mathrm{Au}$ & 1.49204 & 40.50705 & 4.84178 \\
\hline
\end{tabular}


Table S6. The van der Waals radii for the Poisson-Boltzmann solvation calculations.

\begin{tabular}{cc}
\hline $\begin{array}{c}\text { Atomic } \\
\text { symbol }\end{array}$ & $\begin{array}{c}\text { Radii } \\
\text { (in Angstroms) }\end{array}$ \\
\hline \hline $\mathrm{H}$ & 1.150 \\
$\mathrm{C}$ & 1.900 \\
$\mathrm{O}$ & 1.600 \\
$\mathrm{Au}$ & 1.647 \\
\hline
\end{tabular}

Table S7. The binding free energies of $\mathrm{COOH}$ and $\mathrm{CO}$ on the GB-free clean surface using scaled van der Waals radii.

\begin{tabular}{ccc}
\hline Scaling factor & $\Delta \mathrm{G}_{\mathrm{b}}{ }^{\mathrm{CO}}(\mathrm{eV})$ & $\Delta \mathrm{G}_{\mathrm{b}}^{\mathrm{COOH}}(\mathrm{eV})$ \\
\hline \hline 1.0 & -0.397 & -1.420 \\
0.8 & -0.384 & -1.406 \\
0.7 & -0.387 & -1.390 \\
0.6 & -0.380 & -1.359 \\
Reference 8 & -0.310 & -1.310 \\
\hline
\end{tabular}




\section{References}

(1) Soler, J. M.; Artacho, E.; Gale, J. D.; García, A.; Junquera, J.; Ordejón, P.; Sánchez-Portal, D. J. Phys: Condens. Matter 2002, 14, 2745-2779.

(2) Perdew, J. P.; Burke, K.; Ernzerhof, M. Phys. Rev. Lett. 1996, 77, 3865-3868.

(3) Junquera, J.; Paz, Ó.; Sánchez-Portal, D.; Artacho, E. Phys. Rev. B 2001, 64, 235111234119.

(4) Troullier, N.; Martins, J. L. Phys. Rev. B 1991, 43, 1993-2006.

(5) Kobayashi, S.; Takagi, H.; Watanabe, T. Philos. Mag. 2013, 93, 1425-1442.

(6) Rocchia, W.; Alexov, E.; Honig, B. J. Phys. Chem. B 2001, 105, 6507-6514.

(7) Rocchia, W.; Sridharan, S.; Nicholls, A.; Alexov, E.; Chiabrera, A.; Honig, B. J. Comp. Chem. 2002, 23, 128-137.

(8) Lim, H. K.; Shin, H.; Goddard, W. A.; Hwang, Y. J.; Min, B. K.; Kim, H. J. Am. Chem. Soc. 2014, 136, 11355-11361. 